\title{
Spun Fibres for Compensation of PMD: Theory and Characterization
}

\author{
Lynda Cherbi and Abderrahmane Bellil \\ Laboratory of Instrumentation LINS, \\ University of Sciences and Technology Houary Boumedienne, USTHB, \\ Algiers, \\ Algeria
}

\section{Introduction}

The polarization is a relative property to the vibratory nature of light. In an optical fibre, light is a combination of two vibrations of perpendicular directions. Each direction represents one mode of polarization. Indeed, the optical fibres and the components of the optical fibres present a small difference in the refractive index in the pair of the polarization states, a property called the birefringence. This last one induces a difference of propagation speed between the two modes. So, light at the output, cannot be restored more faithfully. The birefringence can change the state of polarization (SOP) of light when it crosses the fibre. In a single mode fibre, the birefringence is combined with a random coupling of polarization modes. The delay measured at the output of the fibre between the two polarization modes is called the difference of group delay DGD (measured in picoseconds). The polarization modes dispersion (PMD) results from the variation of the DGD according to the wavelength and the environment conditions.

The typical tolerance of a system to the PMD is roughly $10 \%$ of the bit period, which gives 40 Ps for a system of $2.5 \mathrm{~Gb} / \mathrm{s}, 10$ Ps for a system of $10 \mathrm{~Gb} / \mathrm{s}$ and only $2.5 \mathrm{Ps}$ for a system of $40 \mathrm{Gbs} / \mathrm{s}$ (Noé et al.,1999). The PMD is a random phenomenon and constitutes an enormous obstacle ahead of the increase of the debits from $10 \mathrm{Gbit} / \mathrm{s}$ for a part of the networks of most telecommunication companies. Several solutions have been proposed to compensate the PMD as: The electronic compensation after a direct photo-detection that can only eliminate a part of the PMD effects since the information about the polarization and the phase get lost at the detection; the second solution is the electronic compensation in a coherent receptor with diversity of polarizations, and the third one is the optical compensation in at least a differential delay section. Other solutions are proposed by the Corning society and which rely on the use of spun fibres allowing the control of the coupling of the modes, therefore reducing the PMD; thus, giving differential group delays of order of Femtoseconds. In the past decade, some considerable efforts have been made to understand the origins of the PMD and to attenuate its effects in the systems. The PMD can be reduced in a fibre with two different manners. The first one consists in minimizing the asymmetries in the refractive index profile and the constraints, which implies improvements of the industrial process in the manufacture of the fibre in order to assure a better geometry and to reduce the rate of constraints in the fiber. The second method allows the control of the coupling of the modes 
of the polarization in the fibre while spinning it during its manufacture. Indeed, the spinning has been used in the manufacture of fibres since the beginning of the 1990s, and it showed that it is an efficient technique to reduce the PMD in the fibre. First, we start by presenting in this work the spun fibres explaining their technology, their principle and their different types. Next, the description of the reduction of the PMD by using the spinning is developed by a mathematical formalism based on the theory of coupling and Jones's matrix. Moreover, the reduction of the PMD is verified in the spun fibres while applying the method of JME and the COTDR method (photon counting -Optical temporal Domain of Reflectometry) that allowed us to measure the DGD of the order of femtosecond (Cherbi et al., 2009). The comparison of the DGD found in this type of new generation of fibres with those of the standard ones, led us to confirm that the spun fibres offer effectively a smaller DGD than those of the standard fibres, emphasizing the importance of this type of fibres in the reduction of the PMD.

We present the different results already published (Cherbi et al., 2009) while using the reflectometers COTDR and POFDR (polarization- Optical Frequency Domain of Reflectometry) which are used to get the polarization characteristics of the spun fibres as the beat length and the PMD and to observe the spatial frequencies linked directly to the period of spinning.

\section{Principle of spun fibres}

\subsection{Technologies of the spun fibres}

There are more than two decades when the concept of the spun fibres has been proposed originally in an article published by (Barlow et al.,1981).

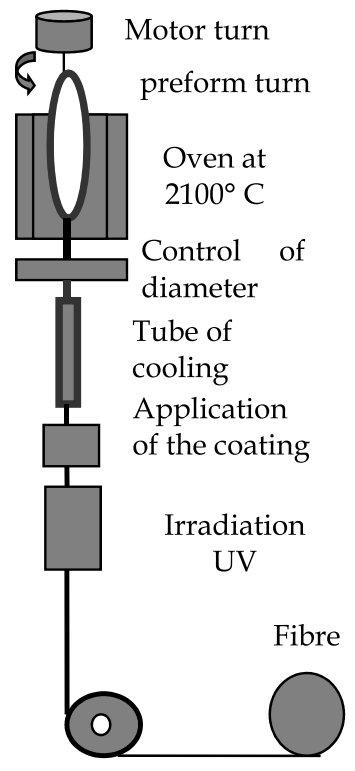

(a)

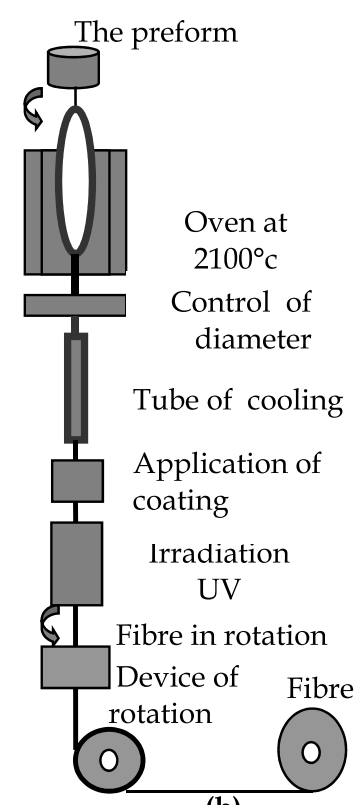

(b)

Fig. 1. Two approaches used to present the rotating fibres (a) turn preforms (b) turn the fibre. 
The fibre spun is achieved by the rotation of the preform during the drawing of the fibre (figure 1.a). In this approach, the system of drawing is the same as that of the conventional standard fibre systems (OVD) except that a rotating motor is placed on the top of the preform. When the motor is set in motion with a predetermined speed, the preform starts turning dragging the rotations of the axes of the birefringence. The rotation will end up with the end of the drawing operation. This approach is quite simple and appropriate for the pulling of the fibre at low speed. However, this is not convenient for the production of the fibre with a high speed pulling because the rotation of the motor must be at very high speed as well. To illustrate this, we consider a rotation rate of the fibre of 3 turns/min, for a drawing speed of $1 \mathrm{~m} / \mathrm{s}$, the rotating speed of the preform is thus only 180 turns/min.

On the other hand, for a modern drawing device having a speed higher than $20 \mathrm{~m} / \mathrm{s}$, the perform must turn at a speed greater than 3600 turns/mn, which is far from practical. For this reason the concept of the spun fibres has not been used in the production of fibres until the half of the nineties when methods of more adapted spinning have been proposed (Ming-Jun \& Nolan, 1998). Moreover, the transmission systems as they appeared at low rate $(<=2.5 \mathrm{~Gb} / \mathrm{s})$, the PMD was not a major problem to seek fibres that perform this reduction.

Several convenient techniques have been suggested during the year 1990, for example, by (Hart et al., 1994] in order to make the fibre turn rather than the preform. Later on, this technique became the most adapted one for the manufacturing of the fibres performing the reduction of the PMD.

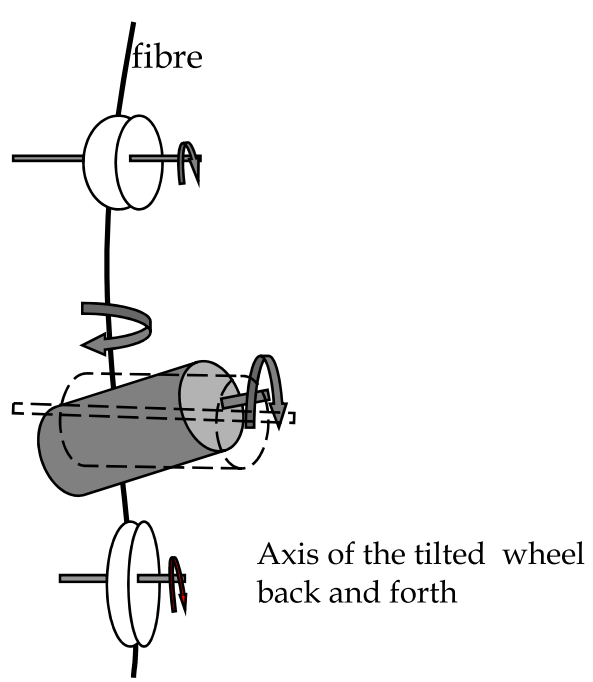

(a)

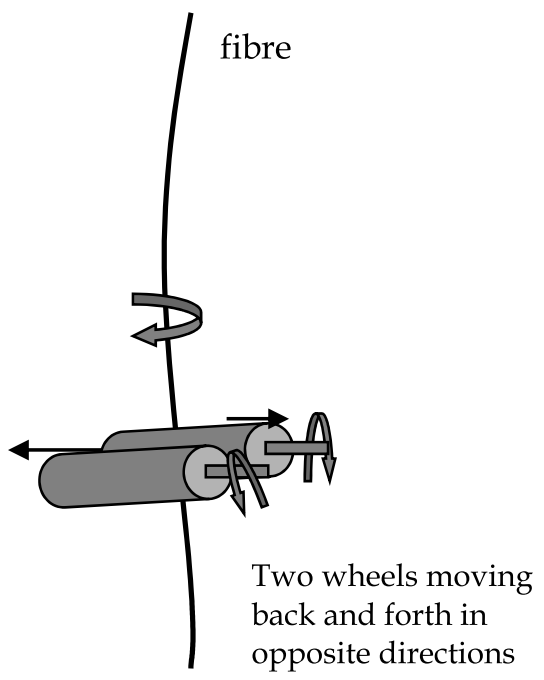

(b)

Fig. 2. Examples of fibre rotation systems: (a) tilted wheel, (b) two wheels moving in opposite directions. 
In this case (fig 1.b), a rotating device of the fibre, is put along the way of fibrage to rotate the fibre directly. Two examples of this device are illustrated in figue 2. In the first example (fig 2.a), a wheel is in contact with the fibre and tilted with respect to its initial position, thus applying a moment of rotation to turn the fibre. In the second example (fig 2.b), two wheels are placed horizontally and are both in contact with the fibre (Blaszyk \& Christoffand,2001). The two wheels move back and forth in opposite directions driving the rotational movement of the fibre. Imposing a direct motion to the fibre eliminates the problem of the preform when turning at high speed. Besides, this technique provides flexibility to control and implement different profiles of rotation for a better reduction of the PMD.

\subsection{Theory of spun fibres}

Two approaches have been suggested to model the reduction of the PMD in the spun fibres, one of which is based on the evolution of the polarization state (Galtarossa et al., 2001; Ming-Jun \&Nolan, 1998). The evolution of the vector representing the polarization dispersion is ruled by the dynamic equation which is linked to the vector of the local birefringence. While solving the dynamic equation, the vector representing the polarization's dispersion is gotten and its module gives the delay of the differential group (DGD). Another approach is based on the theory of the coupled modes of Jones' matrix (Ming-Jun et al., 2002) where the complex amplitudes of the two modes of polarization are described by the equations of the modes coupling. While solving these equations, the complex amplitudes are derived and Jones' matrix is determined and the DGD can be computed from this matrix. Basically, the two approaches give equivalent results. Our survey of the spun fibres is founded on Jones' matrix formalism, where we notice that the analytical solutions obtained are simple.

\subsubsection{Equations of the mode coupling}

As the birefringence in the fibres used in telecommunications is generally small, the formalism based on the theory of disruption (Ming-Jun et al., 2002), can be used to describe the different mechanisms of birefringence in the single mode fibres, including the birefringence due to the distortion of the core, constraints, curvature, rotation of the fibre and torsion. In what follows, we will present the theory of the coupled modes and we will show how to implement it in the different problems of birefringence. Indeed, the small birefringence of telecommunication fibres can be treated as an anisotropic disruption to a material originally isotrope. In the condition of weak guidance, the electric field $\mathrm{E}$ is described by the following wave equation (Dandliker,1992):

$$
\Delta E-\mu_{0} \varepsilon_{0} \varepsilon E=\mu_{0} p
$$

Where $\varepsilon_{0}$ et $\mu_{0}$ are the dielectric and magnetic constants of vacuum respectively, $\varepsilon$ is the relative dielectric constant of the non disrupted fibre, and $\mathrm{p}$ is the disruption term given by the following relation:

$$
p=\varepsilon_{0} \Delta \varepsilon E
$$

Where $\Delta \varepsilon$ is the electric tensor describing the anisotropy of the medium. Without the term of disruption, the equation (1) has modal solutions of the following shape: 


$$
E_{n}(x, y, z)=e_{n}(x, y) \exp \left(-i \beta_{0} z\right) \quad n=1,2
$$

where $e_{n}(x, y)$ is the distribution of the electric field. For a monomode fibre, $\mathrm{n}=1,2$ represent the two modes of polarization.

In absence of disruption, the two modes are degenerated and propagate with the same constant $\beta_{0}$. In presence of the disruption term, it is supposed that the electric field $E(x, y, z)$ is given by the linear superposition of the two non disrupted modes (Ming-Jun et al., 2002):

$$
E(x, y, z)=\sum_{n} A_{n}(z) e_{n}(x, y) \exp \left(-i \beta_{0} z\right)
$$

Where $A_{n}(z)$ are the complex coefficients describing the amplitudes and the phases of the two modes. Let's put equation (4) into equations (1) and (2) and use the relation of orthogonality between the two modes (Ming-Jun et al., 2002):

$$
\int e_{m}(x, y) \cdot e_{n}(x, y) d x d y= \begin{cases}N_{m} & m=n \\ 0 & m \neq n\end{cases}
$$

Knowing that $N_{m}$ is a constant of normalization which can be calculated as follows:

$$
N_{m}=\frac{1}{2} \int \vec{e}_{m} \times \vec{h}_{m}^{*} z d s=\frac{n_{\text {coeur }}}{2}\left(\frac{\varepsilon_{0}}{\mu_{0}}\right)^{1 / 2} \int e_{m}^{2} d s
$$

and using the condition of the weak coupling:

$$
\frac{1}{\beta_{0}}\left|\frac{d^{2} A_{n}}{d z^{2}}\right| \ll\left|\frac{d A_{n}}{d z}\right|
$$

We get the equations of the coupled modes that describe the evolution of the complex amplitudes $A_{n}(z)$ :

$$
\frac{d A}{d z}=i k \cdot A
$$

where $\mathrm{A}$ is the complex amplitude vector taking the following form:

$$
A=\left(\begin{array}{ll}
A_{1} & A_{2}
\end{array}\right)^{T}
$$

and $k$ is the matrix of the coupling coefficients.

$$
k=\left(\begin{array}{ll}
k_{11} & k_{12} \\
k_{21} & k_{22}
\end{array}\right)
$$

The coupling coefficients are associated to the different types of disruptions: 


$$
k_{m n}=\frac{k_{0}}{2 n_{0} N_{0}} \int e_{n}^{*}(x, y) \cdot \Delta \varepsilon(x, y, z) \cdot e_{m}(x, y) d x d y
$$

Where $n_{0}$ is the effective refractive index of both non disrupted modes.

\subsubsection{Jones matrix and the PMD of spun fibre}

The evolution of the local polarization along the birefringent fibre is described by the equations of the modes coupling. The total change of polarization of an input signal, after having traveled a given distance in the fibre is better described by Jones' matrix. Let's assume that the losses in the fibres are negligible, the already predefined Jones matrix, can be put under another form which is:

$$
T=\left[\begin{array}{cc}
A_{1}(z) & -A_{2}^{*}(z) \\
A_{2}(z) & A_{1}^{*}(z)
\end{array}\right] \text { with }\left|A_{1}\right|^{2}+\left|A_{2}\right|^{2}=1
$$

The four complex elements of Jones' matrix can be gotten while integrating the equations of the coupled modes with suitable initial conditions. Once Jones' matrix is known, the PMD can be calculated easily from the elements of the matrix (Chen, 2002; Ming-Jun et al., 2002):

$$
\tau=2 \sqrt{\left|\frac{d A_{1}}{d \omega}\right|^{2}+\left|\frac{d A_{2}}{d \omega}\right|^{2}}
$$

In order to describe the reduction of the PMD, we define a parameter, named reduction factor of PMD (PMDRF) $\zeta$ as the ratio of the DGD of the spun fibres over the DGD of the standard fibre.

$$
\zeta=\frac{\tau}{\tau_{0}}
$$

Where the used lengths for the spun fibres and standard fibres are the same. For example, if $\zeta$ is equal to 1 , the reduction of the PMD is not achieved and if $\zeta$ is equal to 0.5 , a factor of two is obtained in the reduction of the PMD.

\subsection{Different types of spun fibres}

The coupling coefficients matrix depends upon the dielectric tensor of the disruption. The values of these elements are determined by the type of disruption, which means that they depend on the configuration of the fibre. In this section, we describe some configurations of the fibres and we give their coupling coefficients matrix. It is important to note that the coupling matrixes in this work are expressed on the basis of the circular polarization because it is more appropriate to process the rotating fibres (Ming-Jun et al., 2002).

\subsubsection{The linearly birefringent fibre}

The linear birefringence is a consequence of disruptions as the distortions of the core, the asymmetry of the lateral constraints, the curvature. In the case of the linear birefringence, the coupling coefficients matrix is given by (Ming-Jun et al., 2002): 


$$
k=\frac{1}{2}\left(\begin{array}{cc}
0 & \Delta \beta e^{i 2 \varphi} \\
\Delta \beta e^{-i 2 \varphi} & 0
\end{array}\right)
$$

where $\Delta \beta$ is the linear birefringence, and $\Phi$ is the orientation of the birefringence with respect to a given axis

\subsubsection{Spun fibres}

In a spun fiber, the orientation of the birefringence takes place depending on the $x$ axis. The rotation angle $\Phi$ accumulated is therefore a function of the fibre length ' $\mathrm{z}$ ', which in turn is determined by the rate of rotation $\alpha(z)$ :

$$
\Phi=\int_{0}^{z} \alpha(z) d z
$$

Replacing the equation (16) in equation (15), we get the coupling coefficients matrix of the rotating fibres, describing the disruption of the birefringence,

$$
k=\frac{1}{2}\left[\begin{array}{cc}
0 & \Delta \beta e^{i 2 \int_{0}^{z} \alpha(z) d z} \\
\Delta \beta e^{-i 2 \int_{0}^{z} \alpha(z) d z} & 0
\end{array}\right]
$$

\subsubsection{Twisted fibre}

There are two effects in this type of fibres: The rotation of the birefringence and the mechanical torsion. The rotation of the birefringence is similar to that of the rotating fibre. If the rate of torsion is T, the angle $\Phi$ is calculated by

$$
\Phi=T z
$$

The rate of torsion is determined by the coefficients of photo - elasticity of the fibre. The torsion constraint induces the circular birefringence proportionally to the rate of torsion.

$$
\delta=g . T
$$

Where $\mathrm{g}$ is the coefficient determined by the coefficients of photo elasticity of the glass. The typical value of $g$ for fibres in silica is 0.16 . Combining both effects of rotation and torsion, the coupling matrix comes up with the following form:

$$
k=\frac{1}{2}\left[\begin{array}{cc}
\delta & \Delta \beta e^{i 2 T z} \\
\Delta \beta e^{-i T z} & -\delta
\end{array}\right]
$$




\subsection{Solutions of the coupled equations for different types of the spun fibres}

Generally, the matrix of the coupling coefficients depends upon the variable $z$, and the analytic solutions of equation (8) have no existence in the majority of the cases. The numerical integration is always used to get numerical solutions. Different methods, as the method of the finite differences, the Runge-Kutta method (Chen, 2002), can be applied to solve the equation of the coupled modes. However, in the two following special cases, we can derive the analytic solutions which will be discussed in this section.

\subsubsection{Constant spinning rate}

For a constant spinning rate, the function 'spin' (rotation) can be written as follows:

$$
\alpha=\alpha_{0}
$$

Where $\alpha_{0}$ is a constant. In this case, the birefringence of a fibre is estimated in only one direction with a rate $\alpha_{0}$. For this reason, the constant spinning rate is often assigned to an unidirectional spinning. For a spun fibre, with a constant spinning rate, the integral of the coupling matrix can be calculated easily, and the coupled equations become (Hart, 1994):

$$
\begin{aligned}
& \frac{d A_{1}}{d z}=\frac{1}{2} i \Delta \beta e^{i 2 \alpha_{0} z} A_{2} \\
& \frac{d A_{2}}{d z}=\frac{1}{2} i \Delta \beta e^{i 2 \alpha_{0} z} A_{1}
\end{aligned}
$$

With initial conditions $A_{1}(0)=1, A_{2}(0)=0$.

The solutions of equations (22) and (23) are:

$$
\begin{gathered}
A_{1}=-\frac{\alpha_{0}-v}{2 v} e^{i\left(\alpha_{0}+v\right) z}+\frac{\alpha_{0}+v}{2 v} e^{i\left(\alpha_{0}-v\right) z} \\
A_{2}=\frac{\Delta \beta}{4 v} e^{i\left(-\alpha_{0}+v\right) z}-\frac{\Delta \beta}{4 v} e^{-i\left(\alpha_{0}+v\right) z}
\end{gathered}
$$

Where $v=\sqrt{\alpha_{0}^{2}+\frac{1}{4} \Delta \beta^{2}}$

Using equations (12) and (13), we find that the DGD can be expressed by a simple equation for the spinning constant:

$$
\tau(z)=\frac{\gamma_{\omega}}{2 v} \sqrt{(\Delta \beta)^{2} z^{2}+\left(\frac{4 \alpha_{0}}{\Delta \beta} \sin \left(\frac{\Delta \beta z}{2}\right)\right)^{2}}
$$

Where $\gamma_{\omega}=\frac{d \Delta B}{d \omega}=\frac{\tau_{0}}{L}$ is the PMD of a uniform birefringent fibre without modes coupling at the $\mathrm{z}$ position. The sinusoidal term of equation (26) doesn't play an important role when the fibre is sufficiently long. On the other hand, for long fibres, the DGD is given by: 


$$
\tau(z)=\frac{\gamma_{\omega} \cdot \Delta \beta . z}{2 v}
$$

Equation (27) indicates that the DGD progresses linearly with the length of the fibre, and the PMDRF takes the following form:

$$
\zeta=\frac{\Delta \beta}{2 v}
$$

We notice that for spun fibres of constant rate, the PMDRF depends upon the length of beating or the birefringence.

\subsubsection{The periodic spin function}

For the functions of periodic spin, under some conditions, we can describe analytic solutions by using the theory of disruption (Chen et al., 2002) in which fibres are submitted to uniform disruptions only, or in the case of small lengths regime (typically smaller than 100 $\mathrm{m}$ ) in order to fine down their analysis. Indeed, in this approach, the random characteristic of the variation of the disruption in case of important lengths regime is ignored. Using the initial conditions issued from the previous paragraph, the first order solutions of disruption for $\mathrm{A} 1(\mathrm{z})$ and $\mathrm{A} 2(\mathrm{z})$ are as follows:

$$
\begin{gathered}
A_{1}(z)=1 \\
A_{2}(z)=(i / 2) \Delta \beta \int_{0}^{z} \exp \left[-2 i \Theta\left(z^{\prime}\right)\right] d z^{\prime}
\end{gathered}
$$

Where $\Theta(z)=\int_{0}^{z} \alpha\left(z^{\prime}\right) d z^{\prime}$

It becomes easier to obtain the DGD by using equation (13):

$$
\tau(z)=\gamma_{\omega}\left|\int_{0}^{z} \exp \left[-2 i \Theta\left(z^{\prime}\right)^{\prime}\right] d z^{\prime}\right|
$$

Based on the theory of disruption, the first order of the disruption's expansion is valid only when $\Delta \beta<<1$. This condition puts some limits on the application of equation (31) on fibres that have a low PMD.

The validity of this solution has been tested by (chen et al., 2002). When the length of beating is important (some meters), i.e. $\Delta \beta \approx 1$, and the period of spin is smaller than the length of beating, the theory of disruption of the first order can always be applied. For sinusoidal profiles of spin, the expression for the factor of the PMD reduction can be gotten from the solutions of the disruption equation. Let's notice that the profile of sinusoidal spin takes the following form:

$$
\alpha(z)=\alpha_{0} \cos (\eta z)
$$

Where $a 0$ is the spin amplitude, and $\eta$ is the angular frequency of the spatial modulations, which is linked to the spin period $\Lambda$ through the following relationship $\eta=2 \Pi / \Lambda$. 
With the analytical solution of equation (31), we are able to assert which spin parameters give right to the optimization of the PMD performances. With the first observations, we remark that, when the length of beating of a fibre is bigger than some meters, the PMDRF is independent of the beating length, and therefore of the intrinsic birefringence of the fibre. In equation (31), the only contribution to the birefringence of the fibre, comes from $\gamma_{\omega}$, and the DGD is proportional to this size. Let's note that $\gamma_{\omega}$ is the PMD of the unspun fibers (non rotating). On the other hand, the PMDFR will be independent of $\gamma_{\omega}$.

This conclusion is also verified by the direct numerical integration of equation (8) with $\mathrm{k}$ given by equation (17). Some old fibres had beating lengths inferior to some meters; with the improvement of fibre manufacturing, the majority of these lengths were improved lately beyond some meters. The PMDRF independence from the intrinsic birefringence of the fibre, offers the advantage of simplicity in its conception because it is worthless to optimize the spin profiles for the different birefringences of the fibre. Moreover, we noticed that the DGD increases linearly when the length of the fibre increases (figure 3) despite the fact that we got some overlapping oscillations on the graph representing the variation of the DGD with respect to the distance.
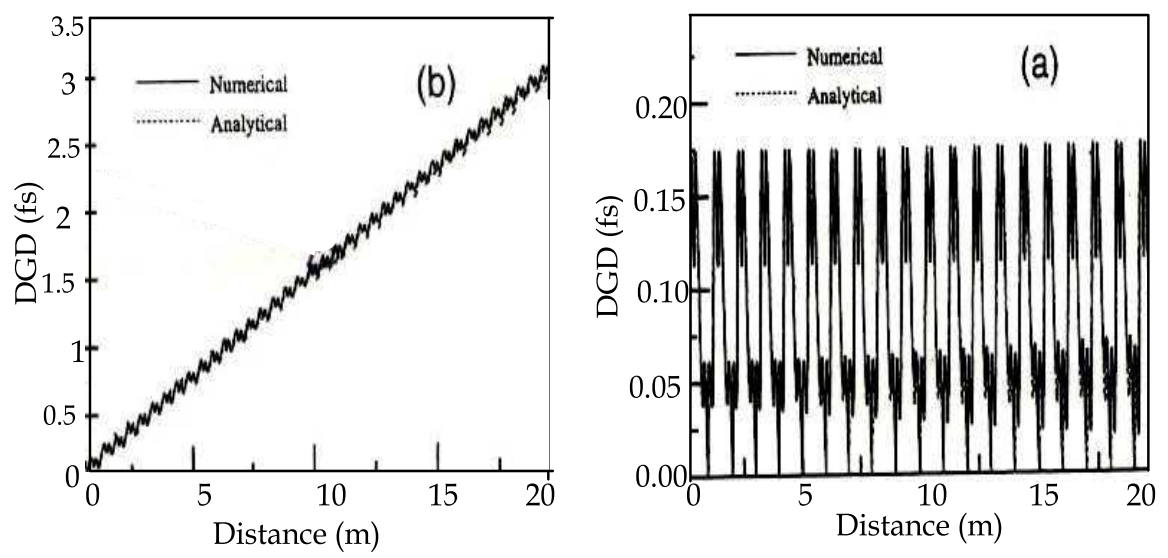

Fig. 3. Evolution of the DGD along a spun fibre.

We can also separate the real and imaginary contributions of the integral (31) in order to better analyze the variation of the DGD along the fibre. We express the equation (31) in an alternative way of the DGD for one spatial period T:

$$
\begin{gathered}
D G D(T)=\gamma_{\omega}\left|\int_{0}^{T} \cos \left[2 \Theta\left(z^{\prime}\right)\right] d z^{\prime}-i \int_{0}^{T} \sin \left[2 \Theta\left(z^{\prime}\right)\right] d z^{\prime}\right| \\
D G D(T)=\gamma_{\omega} \int_{0}^{T} \cos \left[2 \Theta\left(z^{\prime}\right)\right] d z^{\prime}, \quad \Theta(z) \text { is an even function } \\
D G D(T)=\gamma_{\omega} \int_{0}^{T} \sin \left[2 \Theta\left(z^{\prime}\right)\right] d z^{\prime}, \quad \Theta(z) \text { is an odd function }
\end{gathered}
$$


We suppose that $\Theta(z)$ is a periodic function. When $\Phi(z)$ is an even function, $\int_{0}^{T} \sin \left[2 \Theta\left(z^{\prime}\right)\right] d z^{\prime}$ is equal to zero. When $\Theta(z)$ is an odd function, $\int_{0}^{T} \cos \left[2 \Theta\left(z^{\prime}\right)\right] d z^{\prime}$ is equal to zero. For multiple values of the period T, the DGD becomes $n[D G D(T)]$. For values in between, some oscillations are encrusted in the linear variation of the DGD. On the other hand, this survey based on [40] leads us to the conclusion that the dependence of the DGD of the standard fibres on the square root of their lengths, comes from the statistical nature of the random coupling of the two modes of polarization. The linear evolution of the DGD with respect to the length of the spun fibre is caused by the periodicity of the coupling induced by the spinning, thus we have a coupling mode better-controlled than in the case of standard fibres. However, it is possible that the DGD of the spun fibres follows a different evolution law in a region where the first order theory of disruption is not valid any more; for example, when the intrinsic birefringence of the fibre is high and / or the spin rate is high.

With the aforementioned results, it is rather simple to find the phase matching conditions for which the maximum reduction of the PMD can be obtained. In this case, the condition is fixed such that the PMDFR is equal to zero (chen et al., 2002):

$$
\int_{0}^{T} \exp \left[-2 i \Theta\left(z^{\prime}\right)\right] d z^{\prime}=0
$$

Equation (34) can be expressed in another way if we use the properties discussed previously for even and odd functions. We notice that when the phase matching conditions are satisfied, the evolution of the DGD along the spun fibre is periodic. The DGD doesn't increase anymore when the length of the fibre increases.

Equations (31) and (34) are valid for a whole category of periodic profiles of spin. To illustrate the way how to determine the phase matching conditions, we take an example of a sinusoidal spin profile. Such a profile is defined by equation (32). The integration of this profile gives $\Theta(z)=\alpha_{0} \sin (\eta z) / \eta$; then we get the DGD by using equation (31):

$$
\operatorname{DGD}(z)=\gamma_{\omega}\left|\int_{0}^{z} \exp \left[-i \frac{2 \alpha_{0} \sin \left(\eta z^{\prime}\right)}{\eta}\right] d z^{\prime}\right|
$$

The integral can be valued analytically by using the following identity:

$$
\exp [-i x \sin (\theta)]=J_{0}(x)+2 \sum_{n=1}^{\infty} J_{2 n}(x) \cdot \cos (2 n \theta)-2 i \sum_{0}^{\infty} J_{2 n+1}(x) \sin [(2 n+1) \theta]
$$

Then, we get

$$
D G D(z)=\gamma_{\omega}\left[R^{2}(z)+I^{2}(z)\right]^{1 / 2}
$$

where

$$
R(z)=J_{0}\left(2 \alpha_{0} / \eta\right) z+\sum_{n=1}^{\infty} \frac{J_{2 n}\left(2 \alpha_{0} / \eta\right)}{\eta n} \sin (2 n \eta z)
$$




$$
I(z)=\sum_{n=0}^{\infty} \frac{J_{2 n+1}\left(2 \alpha_{0} / \eta\right)}{\eta(2 n+1)} \cos [(2 n+1) \eta z]
$$

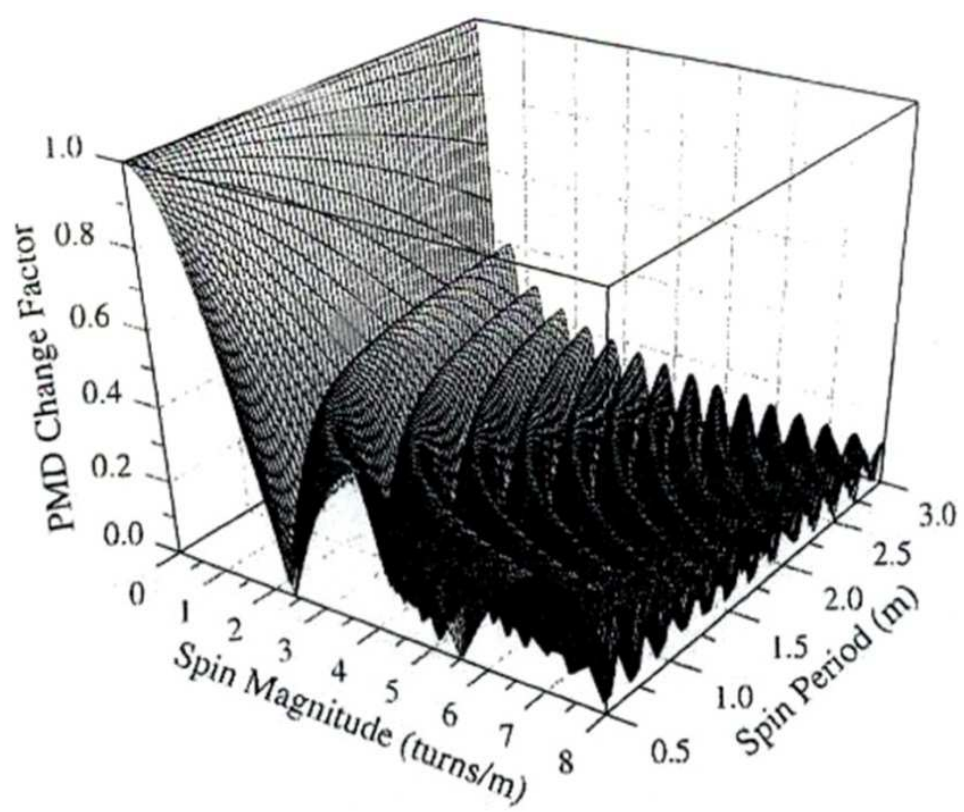

Fig. 4. The PMD change factor as a function of the spin amplitude and the spin period.

We note that when $J_{0}\left(2 \alpha_{0} / \eta\right) \neq 0$, the dominant contribution comes from the term of the linear increase of equation (38). Neglecting the oscillations term, the expression of the DGD becomes:

$$
\operatorname{DGD}(z)=\gamma_{\omega} J_{0}\left(2 \alpha_{0} / \eta\right) z
$$

As in the case of spun fibres with a constant rate, in absence of random disruptions, the DGD increases linearly with the length of the fibre; in contrast with the PMDRF which takes a simpler shape:

$$
P M D R F=J_{0}\left(2 \alpha_{0} / \eta\right)
$$

Equation (41) indicates that the PMDRF is independent from the beating length in the case of spun fibres with sinusoidal profile whose beating lengths are equal to some meters or more. When $J_{0}\left(2 \alpha_{0} / \eta\right)=0$, the linear increase term disappears, and the oscillation terms cannot be neglected any more. In this case, the DGD oscillates between 0 and a maximum value and is independent of the propagating distance. The condition where the minimum of the PMD is reached is called the condition of phase matching (figure 4).

Figure 4 illustrates the presentation in three dimensions for the graph of the PMD reduction as a function of the spin period and the spin amplitude. The phase matching condition can 
be achieved for different spin parameters enabling to get an optimal reduction of PMD; though in general, amplitudes of higher spins give a better reduction of PMD. Figure (3.a) shows the evolution of DGD along the fibre with the phase matching condition. Finally, the maximum of reduction can be reached at the zeroes of the Bessel function of order zero (equation 41).

\subsection{Reduction of the PMD for different profiles of spin}

\subsubsection{The constant spin rate}

While using equation (28), the PMDRF, as function of the spin rate, is represented for different beating lengths in figure 5 ; for a constant spin rate.

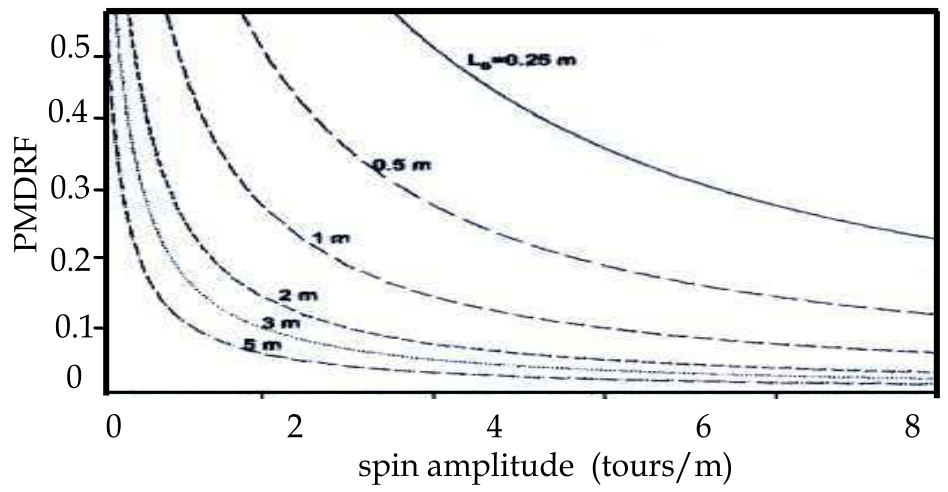

Fig. 5. The factor of reduction of the PMD with respect to the spin amplitude in the case of a constant spinning rate for different beat lengths.

We note that the PMD is reduced when the spin rate increases. For the same spin rate, PMDRF depends on the beating length. The higher the beating length is, the more reduced is the PMD. For a high PMD of the fibre (beating length $<1 \mathrm{~m}$ ), a high spin rate is necessary to reduce the PMD.

\subsubsection{Sinusoidal spin}

In figure 6 , we use a beating length of $1 \mathrm{~m}$ as example to illustrate the reduction of the PMD for sinusoidal types of spin (Ming-Jun et al., 2002).

Figure 6 shows that for sinusoidal spin types, the PMDRF oscillates with the spin amplitude, which is different from the case where the spin was constant. Furthermore, this figure shows that, for a sinusoidal spin, the phase matching condition can be gotten in order to come to a low PMD; on the other hand, in the case of constant spin, the phase matching doesn't exist. The phenomenon of phase matching can be explained by the mechanism of coupling of modes. The constant spin reduces the birefringence of the fibre, and causes no coupling of modes as well. For the sinusoidal spin, the variation in the rate of spin carries along the two modes of polarization to intercouple, reaching a compensation of the PMD. For some spin profile and birefringence of fibre, the conditions of phase matching are satisfied and the maximum of energy exchange occurs in order to provide a better reduction of PMD. The results of modeling indicate that the conditions of phase matching depend on the beating length, the period of the spin and the amplitude of the spin. We can use the same function of 


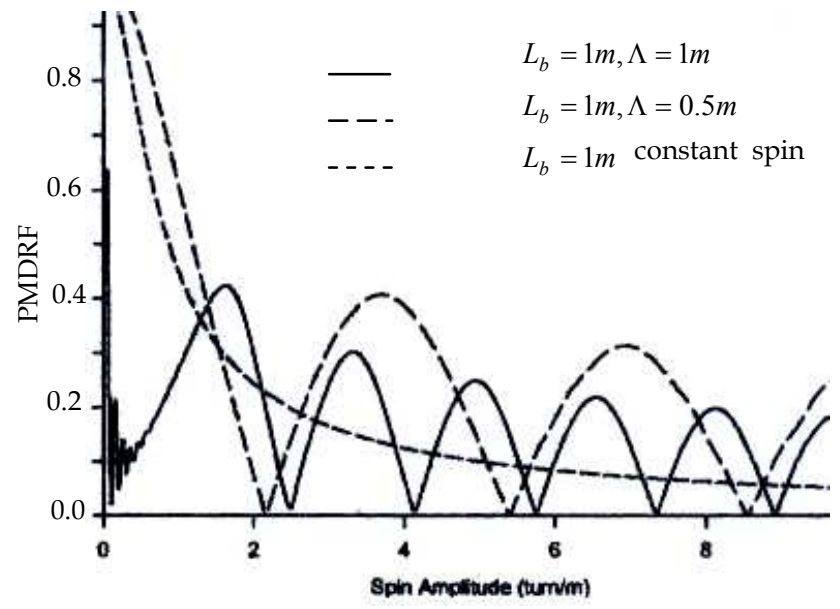

Fig. 6. The factor of reduction of the PMD versus the spin amplitude in the sinusoidal spin profiles.

spin to get a small reduction of PMD for high lengths of beating. However, for small lengths of beating; the phase matching has a strong dependence with the length of beating.

The fact that the birefringence of the real fibres is not constant and changes randomly, it is impossible to have the phase matching for the whole birefringence while using only one sinusoidal spin. This problem can be solved by admitting spin profiles with many Fourier components. To get to this point, the concept of the use of the modulated spin in amplitude and frequency has been developed by the Corning society.

\subsection{Statistical evolution of the PMD of the spun fibres}

As it was mentioned in the previous sections, the spun fibres follow a linear evolution law without the random modes coupling or in the régime of short lengths. When the random mode coupling is present, it has been found that the spun fibres follow an evolution law, a function of square root, similar to that of the unspun fibres, but with a different rate depending on the spin parameters (Chen, 2002). The random mode coupling can be characterized by a random variation of the birefringence axis and / or by the induced phase shift by the external constraints with an occurrence frequency of $1 / h$, where $h$ is called the coupling length of the modes. On the other side, a fibre of length «l» can be divided into $(l / h)$ segments. Using this model, for a sinusoidal profile of spun fibre under no optimal conditions (no phase matching), the DGD can be expressed under the following simple form:

$$
\tau=\zeta \gamma_{\omega} \sqrt{h l}
$$

We notice that the fact that the PMDRF « $\zeta$ » is independent of the beating length when the length of beating is greater than some meters, the DGD in the régime of important lengths, and in presence of the random coupling mode, is corrected by a factor $\zeta$, which is the reduction induced by the fibre spinning during the process of drawing. In this case, the property of evolution of the PMD is similar to that of the fibre possessing the linear birefringence. 


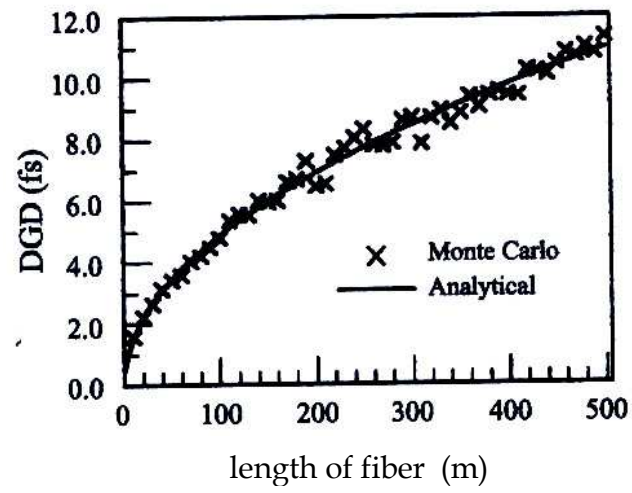

Fig. 7. The DGD of a spun fibre according to the length of the fibre. The amplitude of spin is $3.5 \mathrm{turn} / \mathrm{m}$, the period of spin is $1 \mathrm{~m}$, the length of beating is $10 \mathrm{~m}$ and the length of coupling is $10 \mathrm{~m}$.

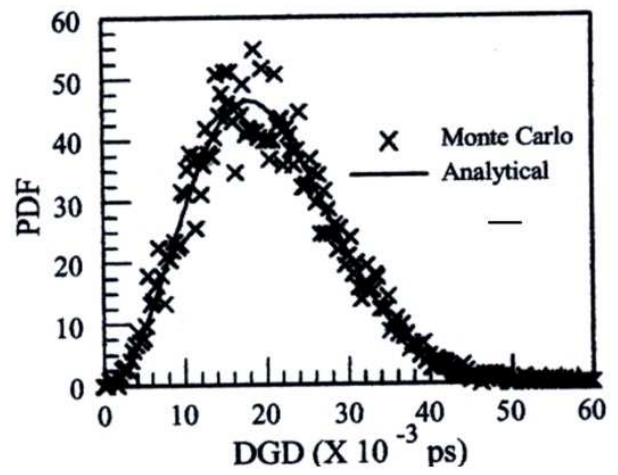

Fig. 8. Probability density function versus the DGD of the fibre when the condition of phase matching is not verified. The amplitude of spin is $3.5 \mathrm{turn} / \mathrm{m}$, the period of spin is $1 \mathrm{~m}$, the length of fibre is $500 \mathrm{~m}$ and the length of coupling is $10 \mathrm{~m}$.

The simplest law of evolution given by equation (42) has been verified by using a numerical modeling (Chen, 2003). Figure 7 shows the results of the numerical simulation for a sinusoidal spun fibre under the non optimal conditions. As it is shown in this figure, the numerical modeling accomodates very well with the theoretical prediction.

In the case of standard fibres (unspun fibres ) with a random coupling mode length $h$, the distribution of the DGD is analog to the distribution of Maxwell, where the standard deviation $\sigma$ used in the expression of PDF can be given by the following expression:

$$
\sigma=\left(\frac{\lambda}{c L_{b}}\right) \sqrt{h \cdot l} / \sqrt{3}
$$

We have proven that the Maxwell distribution is valid in the case of the spun fibres, except that, the parameter $\sigma$ should be corrected by the contribution of the spinning fibre. The modified parameter $\sigma$ is now under the following form 


$$
\sigma=\left[J_{0}\left(2 \alpha_{0} / \eta\right)\right]\left(\frac{\lambda}{c L_{b}}\right) \sqrt{h \cdot l} / \sqrt{3}
$$

This equation has been tested and validated in (chen, 2002). Figure 8 represents the probability density function 'PDF ', according to the DGD of the spun fibre, obtained by numerical calculations and Maxwell distribution equation where we confirm according to the figures that the two results converge perfectly.

When the conditions of phase matching are satisfied, the total DGD of the fibre is a periodic function, and it oscillates between the zero value and a maximal value $\varepsilon_{\max }$. For this reason, the DGD of only one segment of a fibre is linked to the average of DGD inside one period of spin. Therefore, in the regime of high lengths $(l>>h)$, the total DGD can be written as follows (Ming-Jun et al., 2002):

$$
\tau=\varepsilon^{\prime} \varepsilon_{q} \sqrt{l / h}
$$

Where $\varepsilon_{q}$ is the square average of the DGD in one period of spin, and $\varepsilon^{\prime}$ is a coefficient that depends on the average coupling coefficient between two segments. For a condition of phase matching (for example: $\alpha_{0}=2.76$ tours $/ m$ et $\eta=2 \pi m^{-1}$ ), $\varepsilon^{\prime}$ is found equal to 1.194. Besides, the DGD increases when the length of coupling of modes decreases (Ming-Jun et al., 1998). It is foreseeable, because under the conditions of phase matching, the DGD is minimum. Any disruption moves the fibre away from the optimal conditions, implying an increase of the PMD. Despite the fact that the DGD of the optimized spun fibres changes differently with the coupling length in comparison with the DGD of the non optimized spun fibres, the DGD always follows a Maxwell distribution, but with a modified parameter $\sigma$ (Chen, 2002).

$$
\sigma=\left(\varepsilon^{\prime} \varepsilon_{q} \sqrt{l / h}\right) / \sqrt{3}
$$

\section{Application of the JME method for the measurement of the PMD of the spun fibres}

We used the JME method (Derickson, 1998) in order to verify the reduction of the PMD in the spun fibres (Cherbi et al., 2006). This applied method, between 1510 to 1615 nanometers, consists in determining the DGD directly between the two main states of polarization by measuring the Jones matrix of the device under test to a set of wavelengths. In order to determine the PMD of the spun fibres, we take the following steps:

- Measure Jones' matrixes $J M\left(\lambda_{i}\right)$ for a set of wavelengths $\lambda_{1}, \lambda_{2}, \ldots . \lambda_{n}$ of the work range (1510 nm-1615nm)

- $\quad$ Do the product $J M\left(\lambda_{i}+\Delta \lambda\right) \cdot J M^{-1}\left(\lambda_{i}-\Delta \lambda\right)$

- Determine the eigen values $\rho_{1}$ and $\rho_{2}$ of the calculated product of matrix

- $\quad$ The DGD $\left(\lambda_{i}\right)$ is gotten then by (Heffner, 1992):

$$
\operatorname{DGD}\left(\lambda_{i}\right)=\Delta \tau=\left|\frac{\arg \left(\frac{\rho_{1}}{\rho_{2}}\right)}{\Delta \omega}\right|
$$


The PMD of the fibre under test is determined by the arithmetic mean of the ' $n$ ' measured DGD:

$$
P M D=\frac{\sum_{i=0}^{n} D G D\left(\lambda_{i}\right)}{n}
$$

We applied the above procedure to two types of the spun fibres in order to compare their performances. The first fibre is unidirectional of length $212 \mathrm{~m}$ in which we noted that the rotation of the spins was only in one sense once removed it from the spool. On the other hand, for the second bi-directional of length $1 \mathrm{Km}$, the rotation of the spins was in the two senses. The results gotten in the figure 9.a and the figure 9.b, show that this method has a good resolution because it permitted to measure DGD of the order of femtoseconds, and to show that this type of fibre presents effectively low DGD compared to those measured in standard fibres that are of order of the picoseconds. Besides, we noted that the bi-directional fibre possesses a lower DGD than that of the unidirectional one indicating thus the efficiency of the bi-directional spun fibres in the reduction of the PMD.

(a)

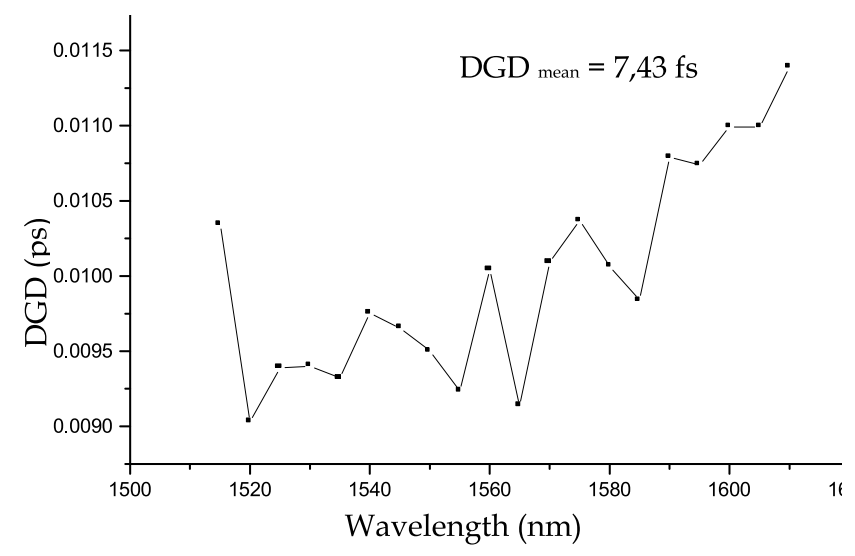

(b)

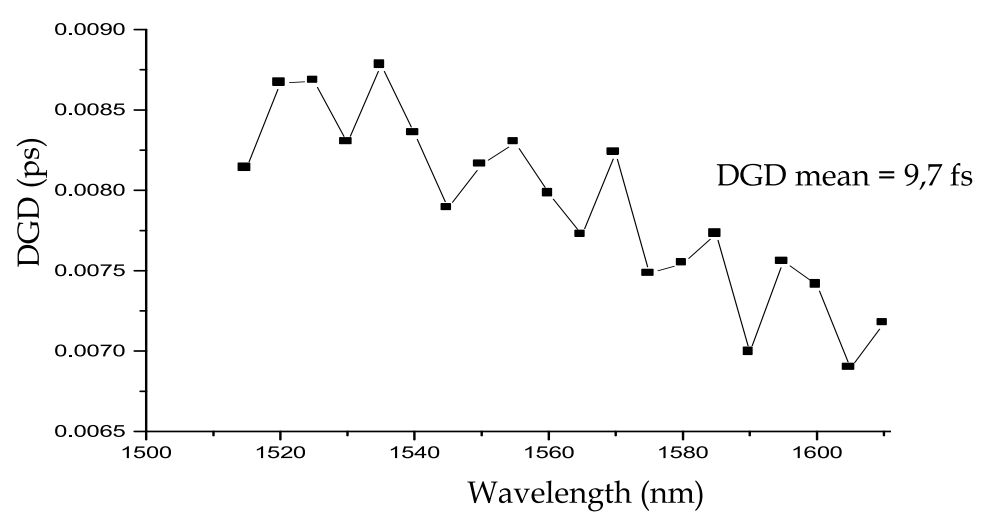

Fig. 9. Representation of DGD measured of spun fibre according to the wavelength for the length (a ) $\mathrm{L}=290 \mathrm{~m}$, (b) $\mathrm{L}=212 \mathrm{~m}$. 
The researchers and inventors of the optical fibre of telecommunication systems predict the impact of PMD from the distribution of $\Delta \tau$, because this results from the variation of $\Delta \tau$ as a function of wavelength and the conditions of the environment. On the other hand, due to this type of variation, the PMD of an optical path is expressed then statistically, as either the average or the root mean square (RMS) of $\Delta \tau\left(\lambda_{i}\right)$ (Derickson, 1998 ). It is interesting to determine the total PMD of a link made of a series of different spun fibres.

For this reason, we took three different lengths of spun fibres (fibre2, 3 and 4). We started by measuring, with the JME method, their PMD separately, for a given temperature while using a reference fibre (fibre1) used in calibration (table 1). Then, we connected the three fibres, and done the measurement of the total PMD in the same experimental conditions. The same procedure has been applied for the two fibres (fibres 3 and 4). We sought for the best relation of computation to determine the total PMD of a link of spun fibres, by testing the two following relations:

$$
\begin{gathered}
P M D_{\text {totale }}=P M D_{1}+P M D_{2}+\ldots \ldots \ldots \ldots . . . P M D_{n} \\
\text { Or } P M D_{\text {totale }}=\sqrt{P M D_{1}^{2}+P M D_{2}^{2}+\ldots \ldots \ldots . . P M D_{n}^{2}}
\end{gathered}
$$

With $n$ the number of fibres used in the link

Our experimental results regrouped in table 1 are in very good agreement with the first relation [Cherbi et al., 2006].

\begin{tabular}{|l|l|l|l|}
\cline { 2 - 4 } \multicolumn{1}{l|}{} & $\begin{array}{l}\text { DGD }(\mathrm{fs}) \text { measured } \\
\text { with the step } \\
(10 \mathrm{~nm})\end{array}$ & $\begin{array}{l}\text { Total DGD }(\mathrm{fs}) \\
\text { calculated with } \\
\text { relation }(48)\end{array}$ & $\begin{array}{l}\text { Total DGD }(\mathrm{fs}) \\
\text { calculated with } \\
\text { relation }(49)\end{array}$ \\
\hline reference fibre of $(1 \mathrm{~km})$ & 98,721 & & \\
\hline Fibre $2(212 \mathrm{~m})$ & 4,8223 & & 13,16 \\
\hline Fibre $3(290 \mathrm{~m})$ & 7,4315 & & 12,25 \\
\hline Fibre $4(1 \mathrm{~km})$ & 9,7399 & 21,99 & \\
\hline connected Fibres $(2+3+4)$ & 22,9437 & 17,17 & \\
\hline connected fibres $(3+4)$ & 17,1985 & & \\
\hline
\end{tabular}

Table 1. The PMD relation of the spun fibres link.

\section{Determination of the polarization's properties of the spun fibres using the reflectometers}

The beat length of the fibre can be measured directly by the extraction of the spatial period of the backscattered signals (Wegmuller, 2002, 2004), which permits to estimate the PMD in the single-mode fibres (Ellison et al., 1998; Chen, 2002). The OFDR method is not exploited again especially for investigating of the spun fibres for the determination of its parameters and of their PMD according to the distance. In this section, we will present the 
relation already demonstrated experimentally by COTDR in our anterior works (Cherbi et al., 2009) existing between the spatial period of the backscattered signal and the PMD of the spun fibre and given by (Chen, 2003). Even more, the COTDR method allowed us to compare the results found with those of the JME method. Afterward we will present the POFDR method which used for spun fibres (Cherbi et al., 2009; Wegmuller et al., 2005) to obtain the beat lengths of the two types of spun fibres and the spin period of the bidirectional fibre.

Chen( Chen, 2003) has demonstrated that the spatial period of the backscattered signals obtained from a POTDR (polarization-sensitive optical time-domain reflectometer) of the spun fibres varies linearly with the beat length of the fibre. This means that for a given beat length, the spatial period $T_{s}$ can be used as calibration for the reduction of the PMD. A simple relation linking the spatial frequency $\mathrm{F}\left(F=1 / T_{s}\right)$ to the beat length and the spin parameters, is given by:

$$
F=\left|J_{0}\left(2 \alpha_{0} / \eta\right)\right| /\left(L_{b} / 2\right)
$$

The PMD of the bi-directional spun fibre is linked to the spatial frequency in the form (Chen, 2003):

$$
P M D=(\lambda / 2 c) F
$$

Thus, the PMD of the bi-directional spun fibres can be determined directly through the measure of the spatial period as in the case of the standard fibres, while measuring the spatial period of the backscattered obtained from the reflectometers. The equation (50) shows that when the spin is zero, the spatial period converges to the one of the standard fibres.

\subsection{Measure of the DGD in the spun fibres by the C-OTDR method}

The technique (COTDR) (Wegmuller et al., 2004) is appropriate to detect the defaults in a given fibre (sites of reflection, losses) with a spatial resolution of the decimetre order. The main difference of this reflectometer (Cherbi et al., 2009) compared to a classic OTDR (Ellison \& Siddiqui, 1998) resides in the use of photon counting detector (InGaAs avalanche photodiode). It is used in the so-called gated Geiger mode, which means that the detector is only active during a short time slot. During this period, only a single photon falls in the detector and triggers an avalanche, which is then detected by electronics discriminator. Contrary to the operation of a classic detector APD in linear regime, this avalanche is no longer proportional to optical input signal power, but independent of it. The detection is therefore a binary one, either there is an avalanche, or not. In order to evaluate the incident optical power (or mean photon number) on the detector during its activation, the detection process (gate opening) must be repeated many times in order to determine the detection probability of photons with a good precision. This probability is proportional to the incident signal power that is smaller than about $40 \%$ (no detector saturation) and larger than the detector thermal noise (dark counts). This condition is satisfied in our set-up by using the variable attenuator before the excitation of the fibre. 
From the detection probability for a certain gate position, set by the delay generator, the reflectivity at the corresponding location in the fibre is readily gotten with a spatial 2- point resolution determined by gate duration. Thus, to have some information on the different positions in the fibre, the gate delay must be adjusted. In our set-up, the user can specify the zoom interval $\left(L_{\text {start }}, L_{\text {stop }}\right)$ for which the reflectivity is automatically measured with a step size (sampling resolution ).

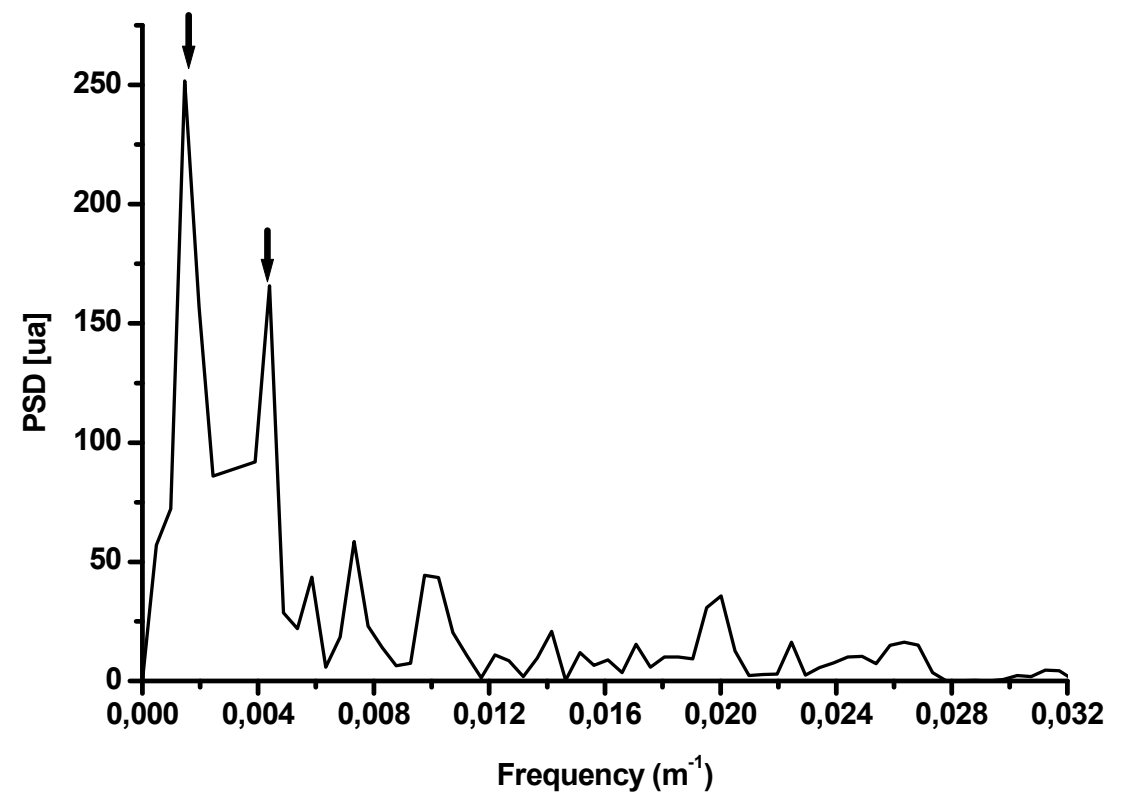

Fig. 10. The Power spectral density of C-OTDR signal for the bi-directional spun fibre of $1 \mathrm{Km}$.

Figure 10 presents the PSD of the backscattered signal power measured by the C-OTDR reflectometer with its spatial frequencies for a bi-directional spun fibre of $1 \mathrm{Km}$ length. Two peaks appear, respectively, at spatial frequencies $\mathrm{F}$ and F/2, in the COTDR trace. A spatial frequency of $0.005 \mathrm{~m}^{-1}$ is gotten from the backscattered signal PSD. The relation (51) gives a 
DGD of the used spun fibre equal to 11.4 femtosecondes. We found for the same fibre a mean value of the DGD equal to 11,53 femtosecondes while using the JME method. These two results are in very good agreement. In conclusion, this demonstrates that the DGD of a spun fibre can be calculated from the spatial frequency of the COTDR signal in accordance with relation (51) permitting to calculate the spatial frequency of a backscattered signal COTDR in a spun fibre from its parameters": $\alpha_{0}=3.5 \pi \mathrm{rad}, \Lambda=1.5 \mathrm{~m}$ and $L_{B}=20 \mathrm{~m}$. The calculated spatial frequency is equal to $F=0.005 \mathrm{~m}^{-1}$, which is equal to the same one measured from the C-OTDR trace. Based on that, we validate the equation, linking the spatial frequency, the spin parameters and the intrinsic birefringence of the bi-directional spun fibre, given in (Chen, 2003)

\subsection{Measure of the beat length of the spun fibres by P-OFDR}

This reflectometer implements the technique of coherent detection sensitive to the polarization in order to get information about the evolution of the polarization states along the fibre under test. In our case, a POFDR is used, implementing the detection of polarization diversity ( Cherbi et al, 2009] and a polarized beam splitter which plays the role of a fixed analyser. The former permits to remove the Rayleigh reflections independent of the polarization by subtracting output 1 from output 2 , thereby removing the frequencies of the back scattered signal that are not related to the fibre birefringence.

The used laser in this reflectometer is a DFB (distributed feedback) characterised by a spectral width of the order of $1 \mathrm{MHz}$ on the whole tuning range, a spatial range of $80 \mathrm{~m}$. Due to the coherent detection, a very good sensitivity of $100 \mathrm{~dB}$ is gotten with this reflectometer. The only factor limiting the resolution of this method is the tuning of the laser. The laser that we used is limited by the continuous tuning of $20 \mathrm{GHz}$ that gives approximately a resolution of $9 \mathrm{~mm}$.

In (cherbi et al., 2009), we have analyzed three types of fibres having the same length of 200 m: a bi-directional spun fibre, a unidirectional spun fibre and a standard fibre. They were wrapped on a table in order to minimize the external constraints. Figure 11 shows the example of the different POFDR traces for different used resolutions of the unidirectional spun fibre (dark line is the mean of different traces). The beat lengths of the two types of spun fibres and the one of the standard fibre are calculated by the following relation (Wegmuller et al., 2002):

$$
<L_{b}>=\frac{1}{s t d(D S P)} \sqrt{\frac{12}{\pi}}
$$

Where PSD is the power spectral density of POFDR signal.

The calculated values of beat lengths derived for the PSD signals of the different fibres: unidirectional spun fibre (figure 11), standard fibre and bi-directional spun fibre (figure 12) are respectively: $50 \mathrm{~m}, 38 \mathrm{~m}$, and $150 \mathrm{~m}$. We note that the beat length of the bi-directional spun fibre is more important than those of the others, which means that the PMD of the bidirectional spun fibre is lower than that of the two other types of fibres, result that we found with the JME method. It also confirms that the bi-directional spun fibre reduce efficiently the PMD compared to the unidirectional spun and the standard fibres. 


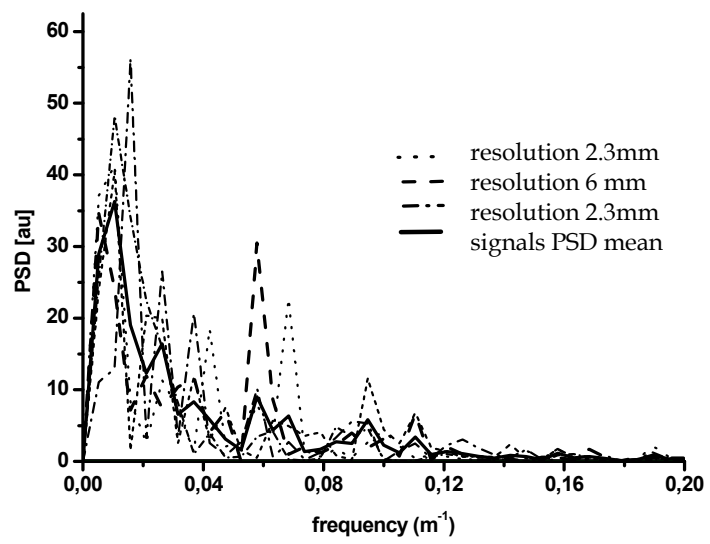

Fig. 11. The mean power spectral density of the backscattered signals POFDR, obtained for different resolutions, of the unidirectional spun fibre of $200 \mathrm{~m}$ length.

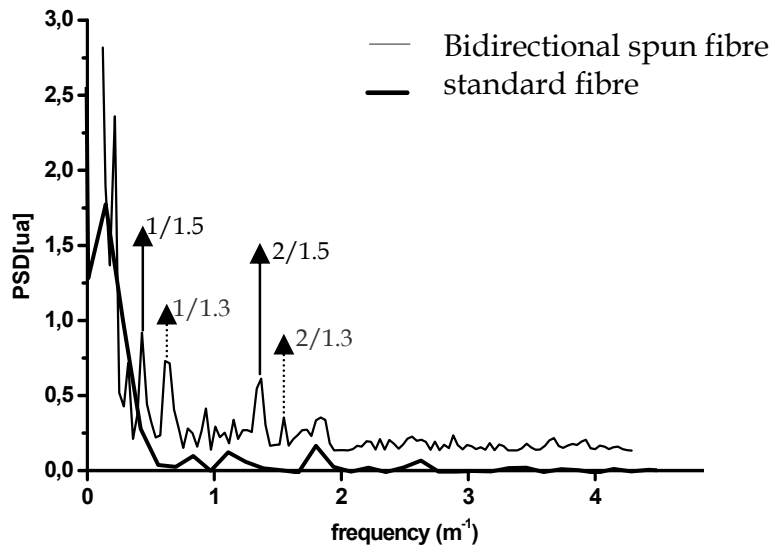

Fig. 12. The mean power spectral density of the backscattered signals POFDR obtained for bi-directional spun fibres and standards of lengths $200 \mathrm{~m}$. 


\section{Conclusion}

In this chapter, we presented the principle of the spun fibres with their technology and their role in the reduction of the PMD in a transmission link of optical fibre. Several types of spun fibres have been given. The theory of these fibres based on the equations of the coupled modes has been detailed.

The reduction of the PMD in these fibres is verified while applying the JME method and the COTDR method used to measure the DGD of the order of femtoseconds. We also confirmed this result while measuring the beat length of these fibres with the POFDR method and compare it with that of a standard fibre. This comparison proved the efficiency of this type of fibres in the reduction of the PMD. Finally, according to the use of the COTDR and POFDR, we concluded that the validity of the data analysis, obtained from the reflectometers and used nowadays for standard fibres, has been demonstrated for the spun fibres and more precisely for the bi-directional spun fibres. Besides, the high spatial resolution of the POFDR enables again the observation of the spatial frequencies directly linked to the spin period, so a precise characterization of the spun fibres can be accomplished.

\section{References}

Barlow, A.J; Ramskov-Hansen; Payne,D.N. birefringence and polarization mode dispersion in spun single mode fibers", applied optics, vol.20 (1981), pp 2963.

Blaszyk, P.E. \& Christoffand, W.R. method and apparatus for introducing controlled spin in optical fibers", U.S patent 63224872 B1 (December 2001).

Chen, X.; Ming, J. Li. \& Nolan, D. A. Polarization mode dispersion of spun fibers : an analytical solution . optics Letters, vol.27(2002),pp. 294 -296.

Chen, X. Scaling properties of polarization mode dispersion of spun fibers in the presence of random mode coupling. optics letters, vol. 27 (2002),pp. 1595.

Chen,X. properties of polarization evolution in spun fibers, optics letters, vol. 28 ( 2003), pp. 2028.

Cherbi,L.; Azrar,A.; Mehenni,M. \&Aksas, R. Characterization of the Polarization in the Spun Fibers. Microwave and Optical Technology Letters, Vol.51, N.2, (February 2009), pp. 341-347, ISSN 0895-2477

Cherbi, L.; Mehenni, M. \& Wegmuller, M. Mesure de la dispersion des modes de la polarisation dans les fibres optiques spun par la méthode d'analyse des valeurs propres de la matrice de Jones . 25 iémes Journées Nationales d'Optique Guidées JNOG, pp 176-178, Metz, France, Novembre 2006.

Dandliker, R (1992). Rotational effects of polarization in optical fibers in anisotropic and nonlinear optical waveguides, C, G. Someda, Elsevier, New york, pp. 39 - 76.

Derickson. D (1998). Fiber optic, test and measurement. Hp professionals books, Prentice hall.

Ellison, G. \& Siddiqui, A. S. A fully polarimetric optical time domain reflectometer", IEEE Photonics technology letters, vol.10 (1998), pp. $246-248$.

Galtarossa, A.; Palmieri, L. \& Pizzinat, A. optimized spinning design for low PMD fibers: an analytical approach. Journal of lightwave technology, vol. 19 (2001), pp. 1502.

Hart, A.; GHuf, R. \& Walker. method of making a fiber having low polarization mode dispersion due to a permanent spin", U.S patent 5, Vol. 47 (1994), pp. 298, March 29. 
Heffner,B. Automated measurement of polarization mode dispersion using Jones matrix eigenanalysis. IEEE Photonics technology Letters, vol.4 (1992), n9, pp.1066.

Ming-Jun,Li \&Nolan, D. fiber spin profile designs for producing fibers with low polarization mode dispersion. optics letters, vol. 23(1998), pp. 1659 - 1661.

Ming-Jun, li; Chen, X. \& Nolas, A. fiber spinning for reducing Polarization mode dispersion in single mode fibers: theory and applications, science and technology division, Corning Incorporated, Sp - AR 02-2 (2002), Corning NY14831.

Noé et al. Polarization mode dispersion compensation at 10, 20, and $40 \mathrm{~Gb} / \mathrm{s}$ with various optical equalizers. IEEE journal of lightwave Technology, vol. 17 (1999), pp 1602 1610.

Wegmuller, M.; Scholder, F. \& Gisin,N. Photon counting OTDR for local birefringence and fault analysis in the metro environment. Journal of lightwave technology, vol. 22 (2004), $\mathrm{N}^{\circ} .2$.

Wegmuller, M.; Legré, M. \& Gisin, N. Distributed Beatlength Measurement in single mode fibers with optical frequency domain reflectometry, journal of light wave technology, vol 20 (2002), N 5, pp. 829.

Wegmuller, M.; Cherbi,L. \& Gisin, N. Investigation of spun fibers using high resolution reflectometry. Proceeding OFC IEEE of Optical Fiber Conference, America 2005. 


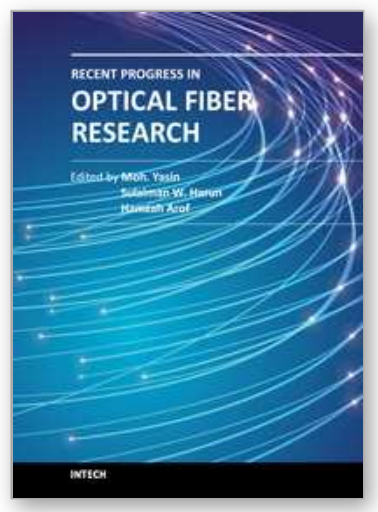

\author{
Recent Progress in Optical Fiber Research \\ Edited by Dr Moh. Yasin
}

ISBN 978-953-307-823-6

Hard cover, 450 pages

Publisher InTech

Published online 25, January, 2012

Published in print edition January, 2012

This book presents a comprehensive account of the recent progress in optical fiber research. It consists of four sections with 20 chapters covering the topics of nonlinear and polarisation effects in optical fibers, photonic crystal fibers and new applications for optical fibers. Section 1 reviews nonlinear effects in optical fibers in terms of theoretical analysis, experiments and applications. Section 2 presents polarization mode dispersion, chromatic dispersion and polarization dependent losses in optical fibers, fiber birefringence effects and spun fibers. Section 3 and 4 cover the topics of photonic crystal fibers and a new trend of optical fiber applications. Edited by three scientists with wide knowledge and experience in the field of fiber optics and photonics, the book brings together leading academics and practitioners in a comprehensive and incisive treatment of the subject. This is an essential point of reference for researchers working and teaching in optical fiber technologies, and for industrial users who need to be aware of current developments in optical fiber research areas.

\title{
How to reference
}

In order to correctly reference this scholarly work, feel free to copy and paste the following:

Lynda Cherbi and Abderrahmane Bellil (2012). Spun Fibres for Compensation of PMD: Theory and Characterization, Recent Progress in Optical Fiber Research, Dr Moh. Yasin (Ed.), ISBN: 978-953-307-823-6, InTech, Available from: http://www.intechopen.com/books/recent-progress-in-optical-fiber-research/spunfibers-for-compensation-of-pmd-theory-and-characterization

\section{INTECH}

open science | open minds

\section{InTech Europe}

University Campus STeP Ri

Slavka Krautzeka 83/A

51000 Rijeka, Croatia

Phone: +385 (51) 770447

Fax: +385 (51) 686166

www.intechopen.com

\section{InTech China}

Unit 405, Office Block, Hotel Equatorial Shanghai

No.65, Yan An Road (West), Shanghai, 200040, China

中国上海市延安西路65号上海国际贵都大饭店办公楼405单元

Phone: +86-21-62489820

Fax: $+86-21-62489821$ 
(C) 2012 The Author(s). Licensee IntechOpen. This is an open access article distributed under the terms of the Creative Commons Attribution 3.0 License, which permits unrestricted use, distribution, and reproduction in any medium, provided the original work is properly cited. 